\title{
The multiple interviewing paths of qualitative health research: what opportunities for neurosciences?
}

\author{
Claudio A. Bosio - Guendalina Graffigna \\ Michela Balconi
}

Faculty of Psychology, Catholic University of Sacreted Heart of Milan, Milan, Italy

guendalina.graffigna@unicatt.it

Qualitative research has for long viewed health as an area of primary interest in which to test its capacity to identify new research topics, to develop theoretical paradigms and methods of inquiry, and to generate applications important for individuals and society (Pope, 1995; Jones, 1995; Grypdonck, 2006; Bosio, 2012). The richness of the results produced is well known and documented (Morse, 2007; Morse, 2010), and it has been confirmed by the remarkable variety of papers presented at the 2nd Global Congress for Qualitative Health Research recently hosted at the Catholic University of Sacreted Heart of Milan (Italy), $28^{\text {th }}-30^{\text {th }}$ of June 2012, of which we will present here a selection.

The reasons which foster this richness of possible applications and methodological solutions of qualitative health research (QHR) can be tracked in several factors.

The first seems to be the well documented ability of QHR to intercept and face problems aroused in the real social context. This is also linked to the applicative nature of qualitative research, which main goal - from the beginning of its epistemological and methodological roots (Glaser \& Strauss, 1967 ) - is to be able not only to cast light on real and up-to-date issues that need to be dealt; but in particular to produce "usable knowledge" able to sustain intervention and to produce change.

The second reason appears to be structural and internal to QHR discipline (Bosio \& Graffigna, 2012): namely linked to the plurality of theoretical and methodological options ascribable to QHR tradition. In particular the multiplicity of interlacement and combination paths among these options valorizes QHR: interlacements that allow a great flexibility and creativity of

Neuropsychological Trends - 12/2012

http://www.ledonline.it/neuropsychologicaltrends/ 
the research design; that make it "ecological" and best suitable to explore real social problems (Richards \& Morse, 2007; Bosio, Graffigna \& Scaratti, 2012).

The third reason relates to QHR ability to dialogue and collaborate with epistemological and methodological traditions apparently and historically far-a-way, but all sharing the same attention to real issues linked to health (e.g. medicine, public health, nursing, neuropsychology, agriculture, engineering). This lively ability to be open to change and debate, to reorient its practice and to invent new ones in order to build bridge between different research communities appear crucial and it is testified both in the literature (Mayan, 2009) as well as in the practice.

As a matter of fact the ongoing vitality and continuous change of the healthcare domain - exemplify for instance from the revision of medicine paradigms in favor of psycho-socio-cultural dimensions of care \& cure, as well as from the post-modern processes of healthcare organizations reconfigurations (Bosio, Graffigna \& Scaratti 2012; Graffigna, Libreri \& Bosio 2012) - require reflection on the appropriateness of traditional research approaches and claims for innovation of methodological paradigms. Thus, at present research perspectives able to bridge different contexts of knowledge and to offer a multifaceted glance on health phenomena are more and more needed. In this frame, qualitative research is receiving an increasing attention, not only as a suitable approach to explore various health issues, but as a possible component of mixed and multi-disciplinary research design. The crossing of research interests and scope between neuropsychological traditions and qualitative methods - as testified in this issue - is only one of the possible collaborative examples in this direction. The proficient joint action between these two domains elucidates the crossing role of the qualitative and quantitative methods viewed as a scientific paradigmatic way to approach health. In this respect, neuropsychology may furnishes a valid test field to verify the sustainability of qualitative practices and the usability of quantitative methods, which, taken together, allow to reconstruct the compound picture of "to be how" and "to intervene how" for care \& cure.

In this special section we exemplify only a few possible directions of the interlacement between qualitative research and other epistemological disciplines and methodological traditions in the health domain: our aim is to testify the potentialities of these experiences in increasing research ability to intercept and read health issues and to produce usable knowledge to generate change and innovation in care \& cure interventions. More into details:

- The paper of Sozzi and Balconi and the paper of Pett and Clark, discuss the continuity and discontinuity of qualitative and quantitative methodological traditions in health research and witness the potentiality of interlacing them in a productive and applicative dialogue. 
- The paper of Leone et al. as well as the paper of Bellardita et al. exemplify the ability of qualitative research to read the intangible aspects of the care \& cure relationship and to explore expert and lay knowledge and meaning making processes intertwines and mismatches, in particular in challenging situation as the care of end of life or as the treatment of prostate cancer.

- The paper of Masako et al. and the paper of Libreri and Graffigna testify the ability of qualitative research to allow an "ecological" and situated glance at the phenomena under investigation, which can be studied during their development.

- The paper of Senes et al. and the paper of Alvarez et al. show how qualitative research can be considered not only a strategy to produce insights and new information regarding one given phenomenon; it can also become the privileged arena in which to develop new approaches and devices (i.e. healing garden practices for neurological rehabilitation; the development of new technological devices for nursing practices in Parkinson care) for the better management of care \& cure.

Although very various in the objects explored and in the methodological definition of the adopted research design, these papers share some common ground:

- all researches described moved from a real social problem and they sought to find the best balance as possible between applicative dimension and sophistication of methods and research design;

- in all cases presented, qualitative research assumed an essential but not dominant role, as main support and counsellor of the whole research process, but often as not unique and autonomous (since often needing the synergy with other - quantitative - approaches). Furthermore qualitative research seemed able to qualify the all research process, enhancing it flexibility and its ductility in aim of a better resonance to the object of the study;

- finally all cases well testify the flexibility of possible qualitative research paths, enhancing the ecological power and creativity of all the research process.

The papers hosted in this special section suggest promising new kind of interlacement between qualitative research and other epistemological as well as methodological traditions in order to produce better usable knowledge and to better sustain change and intervention. Many other interlacement paths are possible, and many more can be invented and seek in the next future! However this special section well witnesses how the situated and engaging nature of qualitative research has the potential to transform research practices into the basis for learning and change in healthcare settings (Heller, 2004). As a matter of fact, qualitative research appears to be the best strategy with which to explore phenomena arising at the intersection among the evolving trends which are reconfiguring both the roles and subjectivity of individuals (i.e. the 
role of patients, of their peer networks and of their lay social contexts) and care \& cure organized practices (in the direction of enhanced complexity and fluidity). Moreover, the joint action with quantitative approach allows to refute a separation between methodologies and practices, in favor of the "best and more useful" for healthcare that points out the renovation of a systems of knowledge.

\section{REFERENCES}

Bosio, A.C. (2012). Editoral. In: Graffigna, G., Morse, J.M., \& Bosio, A.C. (eds.), Engaging people in health promotion and well-being. New opportunities and challenges for qualitative research. Milano: Vita \& Pensiero.

Bosio, A.C., \& Graffigna, G. (2012). "Issue-based research" and "process methodology": reflections on a postgraduate master's program in qualitative methods. Psychology Learning and Teaching, 11 (1), 52-59.

Bosio, A.C., Graffigna, G., \& Scaratti, G. (2012). Knowing, learning and acting in health care organizations and services: challenges and opportunities for qualitative research. Qualitative Research in Organization and Management, 7 (3).

Glaser, B., \& Strauss, A. (1967). Awareness contexts and social interaction. American Sociological Rewiew, 29, 669-679.

Graffigna, G., Libreri, C., \& Bosio, A.C. (2012). Online exchanges among cancer patients and caregivers: constructing and sharing health knowledge about time. Qualitative Research in Organizations and Management, 7 (3).

Grypdonck, M.H.F. (2006). Qualitative health research in the era of evidence-based practice. Qualitative Health Research, 16, 1371-1385.

Heller, F. (2004). Action research and research action: a family of methods. In: Cassell, C., \& Symon, G. (eds.), Essential guide to qualitative methods in organizational research. London: Sage, pp. 349-360.

Jones, J. (1995). Qualitative research: consensus methods for medical and health services research. British Medical Journal, 311-376.

Mayan, M.J. (2009). Essentials in qualitative inquiry. Walnut Creek, CA: Left Coast Press.

Morse, J.M. (2007). What is the domain of qualitative health research? Qualitative Health Research, 17, 715-717.

Morse, J.M. (2010). Editorial: two decades of qualitative health research. Qualitative Health Research, 20, 291-292.

Pope, C. (1995). Qualitative research: reaching the parts other methods cannot reach: an introduction to qualitative methods in health and health services research. British Medical Journal, 31, 42-45.

Richards, L., \& Morse, J.M. (2007). Users guide for qualitative methods, 2nd ed. Thousand Oaks, CA: Sage. 